\title{
Aprendizaje Significativo de Algunos Conceptos Químicos, a través de Resolución de Problemas
}

\author{
Luis Javier Narváez Zamora.
}

Licenciado en Biología y Química. Universidad de Nariño Especialista en Docencia de la Biología. Universidad del Tolima. Maestría en Educación. Instituto de Estudios Superiores de Monterrey. Adscrito al Programa de Licenciatura en Ciencias Naturales y Educación Ambiental, Facultad de Educación de la Universidad Surcolombiana de Neiva. lujanarza@gmail.com

\section{Resumen}

Aprender investigando es una buena alternativa para alcanzar el aprendizaje significativo de conceptos inherentes a las soluciones químicas; los resultados de ésta investigación demuestran que la estrategia constructivista de resolución de problemas, facilita el aprendizaje además de auantificar el grado de construcción cognitiva en estudiantes de Licenciatura en Ciencias Naturales, quienes matricularon el curso de Química General en el primer periodo académico del año 2007.

Metodológicamente se inicia caracterizando el punto de partida conceptual del grupo objeto de estudio, se lo divide en $\mathbf{1 0}$ subgrupos de trabajo y a cada uno se le asigna un problema espećfico de la temática estudiada, el aual debe resolver colaborativamente. Al final se cuantifica el grado de resignificación conceptual; con estos datos y después de establecer la relación de variables, se obtiene un aprendizaje significativo del $77.67 \%$ a partir de las concepciones altemativas del $64.20 \%$ derivadas de experiencias académicas anteriores. El incremento del $13.47 \%$ como ganancia conceptual y la ubicación del $Z$ calculado en la zona de alta significancia de la curva normal, permiten demostrar la eficacia de la resolución de problemas para aprender significativamente la temática abordada.

\section{Palabras clave}

Aprendizaje significativo, resolución de problemas, soluciones químicas.

\section{Abstract}

To leam by investigating is a good altemative to research meaningful learning of inherent concepts to the chemical solutions. The results of this investigation demonstrate that the constructivist strategy of problem solving, facilitates learning as well as quantifying the degree of cognition construction in students of the Licenciatura in Natural Sciences, who signed up for the course of General Chemistry in the first academic period, 2007.

Methodologically, this study commences by characterizing the conceptual starting point of the target group which is subdivided into $\mathbf{1 0}$ subgroups. Each one of the groups is assigned a specific problem of the subject matter studied to be figured out in a collaborative way. In the end, the degree of conceptual resignification is quantified. With these data, and after establishing the relationship between the variables, a meaningful learning of $77.67 \%$ is obtained from the alternative conceptions of $64,20 \%$ derived from previous academic experiences. The increase of $13,47 \%$ as conceptual gain and the location of calculated $Z$ in the zone of high significance of the normal curve, show the effectiveness of the problems to leam the meaningfully approached subject matter.

\section{Key words}

Meaningful leaming, problems resolution, chemical solutions. 


\section{Introducción}

Este trabajo de investigación cuantitativa (Kerlinger y Lee, 2002, citados por Hemández, Fernández y Baptista, 2006; Giroux y Tremblay, 2004), plantea la aplicación de la resolución de problemas como estrategia didáctica investigativa en la búsqueda de aprendizaje significativo (Perales, Álvarez, Fernández, García, González y Rivarrosa, 2000) y (Ausubel, Novak y Hanesian, 1987) de los conceptos inherentes a la temática de soluciones químicas, correspondientes al primer curso de química de la Licenciatura en Ciencias Naturales de la Universidad Surcolombiana de Neiva, Colombia.

El aprendizaje significativo se caracteriza por ligarse con las concepciones alternativas o ideas previas de la estructura cognitiva del aprendiz de manera sustancial y relevante (Ausubel, Novak y Hanesian, 1987) y Ballester, 2002) para ello deben cumplirse cuatro requisitos básicos: la decisión autónoma de aprender, un material y tareas potencialmente significativas, un profesor guía o facilitador del proceso y unas actividades de aplicabilidad de los conceptos construidos (Gil, 1993).

La resolución de problemas a través de la investigación es la estrategia de enlace para propiciar el cambio conceptual, entre las concepciones altemativas del estudiante y el conocimiento cientúfico, tal como lo plantean Posner, Strike, Hewson y Gertzog (1982).

El problema de investigación se plantea bajo la siguiente interrogante-hipótesis: ¿̇a estrategia didáctica constructivista de la resolución de problemas permite construir aprendizaje significativo de los conceptos inherentes a la temática "soluciones químicas"?

\section{Metodología}

La investigación inicialmente se encarga de caracterizar los preconceptos que sobre la temática de soluciones químicas, tiene en su estructura cognitiva un grupo de $\mathbf{3 6}$ estudiantes, matriculados en el primer periodo académico del año 2007 en el aurso de Química General; seguidamente, se forman diez grupos de trabajo y cada uno recibe un problema inherente a la temática objeto de estudio; su resolución le demanda a cada grupo el trabajo colaborativo, a través del cual intentan varias alternativas de solución, para finalmente seleccionar una que se ajuste a las necesidades conceptuales del problema (Ballester, 2002). Resueltos los problemas se caracteriza la estructura cognoscitiva final, ayyos resultados se contrastan estadísticamente con los inidales, para determinar la efectividad y eficacia de la estrategia didáctica seleccionada.

La información se recaba con un cuestionario (anexo 1), donde se seleccionan los conceptos más importantes de las soluciones químicas, previa determinación de su validez y confiabilidad (Cohem, y Swerdlik 2001) y (Aiken, 2003) este instrumento, al igual se sometió a una prueba piloto con el fin de optimizarlo.

La resolución de problemas, implica el seguimiento de nueve pasos fundamentales (Lozano, 2006) y (Perales y otros 2000): definición de aquello que saben los estudiantes de cada grupo de trabajo, definición de aquello que no saben, consulta personal de los conceptos que no saben, socialización de los hallazgos conceptuales, generación de alternativas de solución al problema, escogencia de las opciones de solución más viables, implementación teórico práctica de las alternativas escogidas, elaboración de un informe escrito del tratamiento del problema y socialización de los resultados grupales. 
Una vez conduida la etapa experimental, se procede a caracterizar el estado final de la estructura cognitiva del grupo, empleando un post-test, representado en el mismo cuestionario usado como pretest.

Con los datos sistematizados se efectúa una prueba Z para docimar la hipótesis propuesta.

\section{Resultados}

Estructura cognoscitiva inicial del grupo objeto de estudio Las respuestas otorgadas al pretest por el grupo objeto de estudio se muestran en la tabla 1

Tabla 1 Estructura cognoscitiva inicial del grupo objeto de estudio.

\begin{tabular}{|c|c|c|c|c|c|c|c|c|c|c|c|c|c|c|c|c|c|}
\hline 36 & 5 & 5 & 5 & 5 & 5 & 2 & 5 & 4 & 5 & 5 & 5 & 5 & 5 & 5 & 5 & 4 & 75 \\
\hline EASAJS & 147 & 100 & 127 & 134 & 118 & 99 & 117 & \multicolumn{2}{|c|}{${ }_{125}{ }^{2} \mathrm{MES}_{2}$} & 127 & 100 & 125 & 96 & 105 & 104 & 121 & PQARTAJE \\
\hline$\%$ acierto & 82 & $\frac{2}{56}$ & 31 & 44 & 56 & 55 & 65 & 89 & 57 & 79 & 56 & 69 & 53 & 58 & 58 & 69 & 64.20 \\
\hline 1 & 4 & 2 & 4 & 4 & 4 & 4 & 4 & 4 & 3 & 4 & 4 & 3 & 4 & 2 & 2 & 4 & 56 \\
\hline 2 & 4 & 2 & 2 & 5 & 3 & 2 & 2 & 3 & 2 & 4 & 2 & 4 & 1 & 4 & 3 & 4 & 47 \\
\hline 3 & 5 & 2 & 2 & 3 & 3 & 4 & 3 & 3 & 3 & 4 & 2 & 4 & 4 & 3 & 4 & 3 & 52 \\
\hline 4 & 4 & 2 & 2 & 4 & 4 & 3 & 3 & 5 & 4 & 4 & 3 & 2 & 2 & 3 & 2 & 4 & 51 \\
\hline 5 & 4 & 2 & 2 & 4 & 4 & 3 & 5 & 3 & 3 & 5 & 2 & 2 & 2 & 2 & 4 & 3 & 50 \\
\hline 6 & 4 & 1 & 4 & 1 & 4 & 4 & 3 & 3 & 2 & 2 & 2 & 5 & 3 & 4 & 4 & 3 & 49 \\
\hline 7 & 5 & 4 & 4 & 4 & 2 & 3 & 3 & 3 & 2 & 4 & 5 & 4 & 2 & 3 & 3 & 4 & 55 \\
\hline 8 & 4 & 4 & 3 & 4 & 4 & 2 & 4 & 3 & 3 & 4 & 4 & 4 & 3 & 3 & 2 & 5 & 56 \\
\hline 9 & 5 & 2 & 5 & 5 & 4 & 3 & 2 & 3 & 1 & 4 & 2 & 4 & 2 & 4 & 2 & 3 & 51 \\
\hline 10 & 4 & 2 & 5 & 4 & 4 & 3 & 4 & 5 & 2 & 5 & 4 & 2 & 3 & 3 & 4 & 4 & 58 \\
\hline 11 & 4 & 5 & 1 & 4 & 3 & 3 & 4 & 5 & 3 & 2 & 2 & 4 & 3 & 3 & 2 & 4 & 52 \\
\hline 12 & 4 & 2 & 4 & 4 & 5 & 3 & 4 & 4 & 3 & 2 & 4 & 4 & 2 & 3 & 3 & 3 & 54 \\
\hline 13 & 4 & 4 & 5 & 3 & 4 & 4 & 3 & 5 & 4 & 4 & 2 & 4 & 2 & 2 & 2 & 3 & 55 \\
\hline 14 & 4 & 3 & 4 & 5 & 2 & 3 & 3 & 5 & 3 & 4 & 3 & 4 & 2 & 3 & 3 & 4 & 55 \\
\hline 15 & 5 & 2 & 5 & 3 & 2 & 3 & 4 & 2 & 2 & 5 & 2 & 4 & 4 & 2 & 4 & 5 & 54 \\
\hline 16 & 5 & 4 & 4 & 4 & 5 & 3 & 5 & 3 & 3 & 4 & 4 & 5 & 2 & 3 & 5 & 3 & 62 \\
\hline 17 & 5 & 2 & 3 & 5 & 1 & 2 & 2 & 3 & 2 & 2 & 3 & 5 & 4 & 3 & 2 & 1 & 45 \\
\hline 18 & 5 & 2 & 5 & 5 & 4 & 3 & 3 & 5 & 3 & 4 & 2 & 4 & 1 & 3 & 1 & 2 & 52 \\
\hline 19 & 4 & 2 & 5 & 5 & 4 & 3 & 3 & 5 & 3 & 4 & 4 & 2 & 3 & 3 & 1 & 2 & 53 \\
\hline 20 & 5 & 4 & 4 & 3 & 4 & 3 & 3 & 5 & 3 & 5 & 2 & 4 & 3 & 3 & 4 & 4 & 59 \\
\hline 21 & 4 & 3 & 2 & 4 & 5 & 4 & 3 & 3 & 3 & 5 & 5 & 4 & 3 & 3 & 4 & 3 & 58 \\
\hline 22 & 5 & 1 & 4 & 4 & 1 & 2 & 3 & 3 & 2 & 5 & 2 & 3 & 3 & 4 & 1 & 4 & 47 \\
\hline 23 & 4 & 2 & 2 & 5 & 5 & 3 & 3 & 4 & 2 & 4 & 2 & 3 & 2 & 4 & 5 & 3 & 53 \\
\hline 24 & 5 & 4 & 5 & 5 & 5 & 3 & 4 & 3 & 3 & 2 & 2 & 4 & 3 & 2 & 4 & 4 & 58 \\
\hline 25 & 5 & 2 & 4 & 5 & 2 & 3 & 4 & 4 & 3 & 5 & 3 & 5 & 2 & 3 & 4 & 3 & 57 \\
\hline 26 & 4 & 3 & 5 & 5 & 5 & 2 & 2 & 5 & 3 & 5 & 2 & 4 & 4 & 4 & 5 & 5 & 63 \\
\hline 27 & 5 & 3 & 3 & 1 & 2 & 3 & 5 & 4 & 5 & 5 & 3 & 5 & 1 & 5 & 3 & 5 & 58 \\
\hline 28 & 2 & 3 & 2 & 4 & 2 & 2 & 3 & 1 & 3 & 1 & 2 & 2 & 3 & 3 & 2 & 2 & 37 \\
\hline 29 & 3 & 4 & 3 & 2 & 3 & 1 & 2 & 3 & 2 & 3 & 4 & 2 & 3 & 3 & 2 & 2 & 42 \\
\hline 30 & 2 & 2 & 3 & 4 & 2 & 2 & 3 & 2 & 3 & 1 & 2 & 3 & 2 & 2 & 2 & 3 & 38 \\
\hline 31 & 4 & 3 & 3 & 4 & 3 & 2 & 3 & 3 & 4 & 3 & 4 & 4 & 2 & 1 & 2 & 3 & 48 \\
\hline 32 & 2 & 3 & 4 & 2 & 2 & 2 & 3 & 4 & 3 & 3 & 1 & 2 & 3 & 2 & 2 & 2 & 40 \\
\hline 33 & 2 & 3 & 2 & 1 & 2 & 1 & 2 & 1 & 2 & 1 & 1 & 1 & 2 & 2 & 2 & 3 & 28 \\
\hline 34 & 3 & 2 & 4 & 2 & 3 & 4 & 2 & 2 & 3 & 1 & 2 & 2 & 3 & 2 & 2 & 3 & 40 \\
\hline 35 & 4 & 4 & 3 & 2 & 3 & 2 & 3 & 2 & 2 & 2 & 2 & 2 & 3 & 1 & 2 & 4 & 41 \\
\hline
\end{tabular}


A partir de los datos de la tabla 1 se pueden esbozar los siguientes resultados:

$>$ Los conceptos mejor definidos por el grupo son solución y la solubilidad de las moléculas polares, con un $82 \%$ y un $74 \%$ de acierto respectivamente.

$>$ Por su parte, los conceptos con mayor grado de dificultad en su definición son los de grado de basicidad en función de la concentración molar de hidrogeniónes, grado de solubilidad en función de la naturaleza química de los compuestos, partes por millón y soluto, con grado de acierto del $53 \%$, 55\% $56 \%$ y $56 \%$ respectivamente.

$>$ El promedio de significatividad logrado por el grupo supera en 24.20 puntos al promedio teórico de la prueba. (El máximo puntaje esperado para los 16 reactivos del instrumento 1 es de 80 puntos, calificando con 5 puntos cada reactivo contestado acertadamente).

El grado de aprendizaje logrado por los estudiantes del grupo de estudio para cada concepto se muestra en la gráfica 1.

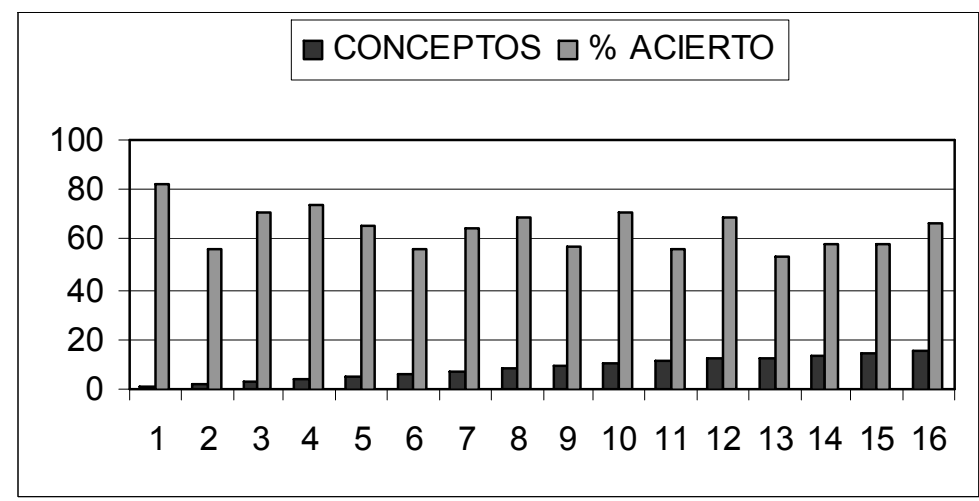

Gráfica 1 Grado de significación inicial de los conceptos objeto de estudio.

En general, el grupo posee como punto de partida conceptual un $64.20 \%$ de aprendizaje significativo, el aual es producto de su experiencia cotidiana pasada, con el cual abordó la definición de los 16 conceptos seleccionados en el anexo 1.

Las medidas de tendencia central correspondientes al pretest se muestran en la tabla 2.

\begin{tabular}{|l|l|l|l|l|l|l|l|}
\hline $\begin{array}{l}\text { DESVIACIÓN } \\
\text { STANDAR }\end{array}$ & VARIANEA & MEDIA & MODA & MÁXIMO & MÍNIMO & $\begin{array}{c}\text { PUNTAJE } \\
\text { TOTAL }\end{array}$ & $\begin{array}{c}\text { \% } \\
\text { ACIERTO }\end{array}$ \\
\hline $\mathbf{8 . 6 8}$ & $\mathbf{7 5 . 3 0}$ & $\mathbf{5 1 . 3 1}$ & $\mathbf{5 6}$ & $\mathbf{7 5}$ & $\mathbf{2 8}$ & $\mathbf{1 8 4 7}$ & $\mathbf{6 4 . 2 0}$ \\
\hline
\end{tabular}

Tabla 2 Medidas de tendencia central del pre-test

El grupo obtiene 1847 puntos de 2880 posibles, es decir, posee un grado de significación del $64.20 \%$ frente a los conceptos del pre-test, situación que revela un conocimiento aceptable sobre soluciones químicas.

Estructura cognoscitiva final del grupo objeto de estudio Las respuestas otorgadas al post-test, se muestran en la tabla 3. 


\begin{tabular}{|c|c|c|c|c|c|c|c|c|c|c|c|c|c|c|c|c|c|}
\hline \multirow{2}{*}{ CASOS } & \multicolumn{16}{|c|}{ ITEMES } & \multirow{2}{*}{ PUNTAJE } \\
\hline & 1 & 2 & 3 & 4 & 5 & 6 & 7 & 8 & 9 & 10 & 11 & 12 & 13 & 14 & 15 & 16 & \\
\hline 1 & 5 & 4 & 5 & 5 & 5 & 2 & 5 & 5 & 4 & 4 & 2 & 4 & 4 & 5 & 4 & 3 & 66 \\
\hline 2 & 4 & 4 & 3 & 4 & 4 & 4 & 5 & 5 & 4 & 2 & 4 & 4 & 4 & 2 & 3 & 4 & 60 \\
\hline 3 & 5 & 4 & 4 & 5 & 5 & 3 & 5 & 4 & 3 & 4 & 2 & 4 & 3 & 2 & 4 & 4 & 61 \\
\hline 4 & 4 & 4 & 4 & 2 & 4 & 3 & 5 & 3 & 1 & 3 & 3 & 2 & 2 & 2 & 3 & 4 & 49 \\
\hline 5 & 5 & 4 & 5 & 4 & 4 & 4 & 4 & 5 & 4 & 4 & 2 & 3 & 2 & 4 & 4 & 3 & 61 \\
\hline 6 & 4 & 4 & 5 & 5 & 5 & 3 & 5 & 3 & 2 & 5 & 2 & 5 & 4 & 4 & 4 & 3 & 63 \\
\hline 7 & 4 & 4 & 4 & 5 & 5 & 2 & 5 & 5 & 4 & 5 & 2 & 4 & 4 & 4 & 4 & 4 & 65 \\
\hline 8 & 4 & 5 & 4 & 5 & 5 & 2 & 4 & 2 & 4 & 2 & 3 & 4 & 4 & 2 & 5 & 5 & 60 \\
\hline 9 & 5 & 4 & 5 & 5 & 5 & 4 & 5 & 3 & 4 & 5 & 1 & 5 & 5 & 5 & 2 & 3 & 66 \\
\hline 10 & 4 & 4 & 5 & 5 & 5 & 2 & 5 & 5 & 4 & 4 & 2 & 2 & 5 & 5 & 4 & 3 & 64 \\
\hline 11 & 5 & 2 & 2 & 4 & 4 & 4 & 4 & 3 & 2 & 5 & 2 & 5 & 2 & 2 & 2 & 4 & 52 \\
\hline 12 & 2 & 4 & 4 & 4 & 4 & 3 & 4 & 4 & 2 & 4 & 4 & 4 & 4 & 4 & 4 & 3 & 58 \\
\hline 13 & 4 & 4 & 5 & 5 & 5 & 3 & 5 & 5 & 3 & 4 & 4 & 3 & 4 & 4 & 4 & 3 & 65 \\
\hline 14 & 5 & 4 & 4 & 5 & 5 & 3 & 5 & 4 & 3 & 4 & 3 & 4 & 5 & 3 & 4 & 4 & 65 \\
\hline 15 & 5 & 5 & 5 & 4 & 4 & 4 & 5 & 4 & 3 & 4 & 4 & 4 & 3 & 4 & 3 & 4 & 65 \\
\hline 16 & 5 & 4 & 5 & 5 & 5 & 4 & 5 & 5 & 4 & 4 & 4 & 2 & 5 & 5 & 5 & 4 & 71 \\
\hline 17 & 5 & 4 & 4 & 4 & 5 & 3 & 5 & 3 & 4 & 4 & 5 & 2 & 5 & 5 & 2 & 4 & 64 \\
\hline 18 & 5 & 2 & 5 & 5 & 4 & 2 & 5 & 4 & 4 & 4 & 2 & 4 & 2 & 4 & 4 & 3 & 59 \\
\hline 19 & 4 & 4 & 4 & 4 & 5 & 2 & 5 & 4 & 3 & 4 & 4 & 4 & 4 & 3 & 4 & 3 & 61 \\
\hline 20 & 4 & 5 & 5 & 4 & 5 & 4 & 5 & 5 & 2 & 4 & 2 & 4 & 2 & 3 & 2 & 4 & 60 \\
\hline 21 & 5 & 4 & 4 & 4 & 4 & 2 & 2 & 3 & 4 & 3 & 4 & 2 & 4 & 4 & 2 & 3 & 54 \\
\hline 22 & 5 & 4 & 4 & 5 & 5 & 3 & 5 & 4 & 4 & 5 & 5 & 2 & 1 & 3 & 2 & 4 & 61 \\
\hline 23 & 4 & 4 & 5 & 5 & 5 & 3 & 5 & 5 & 1 & 4 & 2 & 5 & 5 & 1 & 4 & 3 & 61 \\
\hline 24 & 5 & 4 & 4 & 5 & 5 & 4 & 5 & 5 & 3 & 4 & 2 & 2 & 2 & 3 & 4 & 5 & 62 \\
\hline 25 & 5 & 5 & 5 & 5 & 5 & 5 & 5 & 4 & 5 & 4 & 5 & 5 & 4 & 4 & 5 & 5 & 76 \\
\hline 26 & 5 & 4 & 5 & 5 & 5 & 1 & 5 & 5 & 5 & 5 & 1 & 4 & 5 & 5 & 5 & 5 & 70 \\
\hline 27 & 5 & 5 & 5 & 5 & 5 & 1 & 5 & 2 & 5 & 5 & 1 & 4 & 5 & 5 & 5 & 4 & 67 \\
\hline 28 & 4 & 2 & 5 & 5 & 5 & 4 & 4 & 5 & 2 & 4 & 3 & 4 & 4 & 2 & 4 & 3 & 60 \\
\hline 29 & 5 & 5 & 5 & 3 & 4 & 4 & 5 & 4 & 5 & 4 & 3 & 2 & 1 & 5 & 4 & 4 & 63 \\
\hline 30 & 4 & 4 & 5 & 5 & 6 & 3 & 2 & 3 & 3 & 5 & 2 & 5 & 2 & 3 & 1 & 4 & 57 \\
\hline 31 & 5 & 4 & 4 & 4 & 5 & 2 & 5 & 4 & 1 & 5 & 2 & 4 & 5 & 2 & 2 & 2 & 56 \\
\hline 32 & 4 & 2 & 4 & 2 & 1 & 2 & 5 & 7 & 4 & 4 & 2 & 2 & 5 & 4 & 5 & 4 & 57 \\
\hline 33 & 4 & 4 & 4 & 4 & 4 & 4 & 2 & 4 & 5 & 4 & 2 & 4 & 5 & 4 & 4 & 4 & 62 \\
\hline 34 & 4 & 4 & 4 & 4 & 4 & 3 & 4 & 3 & 5 & 3 & 2 & 4 & 2 & 2 & 3 & 5 & 56 \\
\hline 35 & 5 & 4 & 4 & 4 & 5 & 3 & 4 & 5 & 4 & 4 & 2 & 4 & 3 & 4 & 5 & 4 & 64 \\
\hline 36 & 5 & 5 & 5 & 5 & 5 & 5 & 5 & 2 & 5 & 5 & 5 & 5 & 5 & 5 & 5 & 4 & 76 \\
\hline TOTAL & 162 & 143 & 159 & 159 & 166 & 110 & 164 & 146 & 125 & 147 & 100 & 131 & 131 & 128 & 131 & 135 & 2237 \\
\hline $\begin{array}{l}\% \text { de } \\
\text { acierto }\end{array}$ & 90 & 79 & 88 & 88 & 92 & 61 & 91 & 81 & 69 & 82 & 56 & 73 & 73 & 71 & 73 & 75 & 77.67 \\
\hline
\end{tabular}

Tabla 3. Estructura cognoscitiva final del grupo objeto de estudio.

A partir de los datos pertenecientes a la tabla 3 se pueden esbozar los siguientes resultados:

$>$ Los conceptos mejor definidos por el grupo son: p.p.m. y pH, con un $92 \%$ y un $91 \%$ de acierto respectivamente.

$>$ Por su parte, el concepto con mayor grado de dificultad en su definición es el de dillución, con grado de acierto del $56 \%$ 
D El grupo en general alcanza 37.67 puntos por encima del promedio teórico de 80 puntos posibles para el instrumento evaluativo empleado.

$>$ El grado de aprendizaje logrado por los estudiantes del grupo de estudio para cada concepto se muestra en la gráfica 2

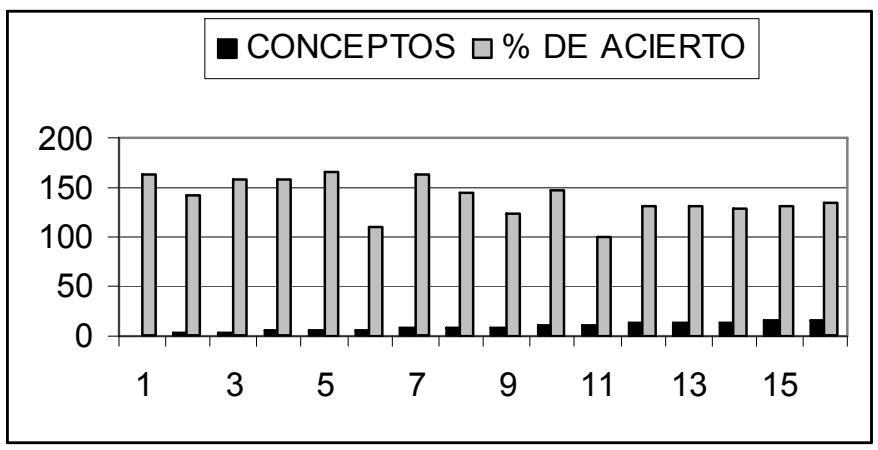

Gráfica.2 Grado de significación final de los conceptos objeto de estudio.

El grupo objeto de estudio en general, alcanza un punto de llegada conceptual del $77.67 \%$ de aprendizaje significativo, el cual es producto de la interacción de su experiencia cotidiana pasada 0 preconceptos y la estrategia didáctica de resolución de problemas en la búsqueda de aprendizaje significativo de los 16 conceptos abordados en el estudio.

Por su parte, las medidas de tendencia central correspondientes al post-test se muestran en la tabla 4.

\begin{tabular}{|l|l|l|l|l|l|l|l|}
\hline $\begin{array}{l}\text { DESYIACIÓN } \\
\text { STÁNDAR }\end{array}$ & VARTANZA & MEDIA & MODA & MÁXIMO & MÍNIMO & $\begin{array}{l}\text { PUNTAJE } \\
\text { TOTAL }\end{array}$ & $\begin{array}{l}\text { \% DE } \\
\text { ACIERTO }\end{array}$ \\
\hline 5.72 & $\mathbf{3 2 . 6 9}$ & $\mathbf{6 2 . 1 4}$ & $\mathbf{6 1}$ & $\mathbf{7 6}$ & $\mathbf{4 9}$ & $\mathbf{2 2 3 7}$ & $\mathbf{7 7 . 6 7}$ \\
\hline
\end{tabular}

Tabla 4. Medidas de tendencia central del post-test

El grupo alcanza 2237 puntos de 2880 posibles, es decir, que finaliza con un grado de significación del $77.67 \%$ frente a los conceptos del post-test, situación que revela un mejoramiento conceptual total del $13.47 \%$ q de la temática estudiada.

Discusión de los resultados obtenidos

Es muy importante enfatizar la eficacia de la estrategia didáctica empleada, pues si bien el $13.47 \%$ de resignificación logrado no parece ser relevante; debe notarse que el grupo, en general tiene un notable $64.20 \%$ de preconceptos muy cercanos a las definiciones que actualmente son aceptadas por la comunidad académica.

Para evidenciar esta situación, en la gráfica 3 se muestra la diferencia de la estructura cognitiva del grupo objeto de estudio, la cual es producto de la implementación de la estrategia constructivista de la resolución de problemas, como un mecanismo del enfoque de aprendizaje por investigación dirigida, (Pozo y Gómez Crespo, 2004). 


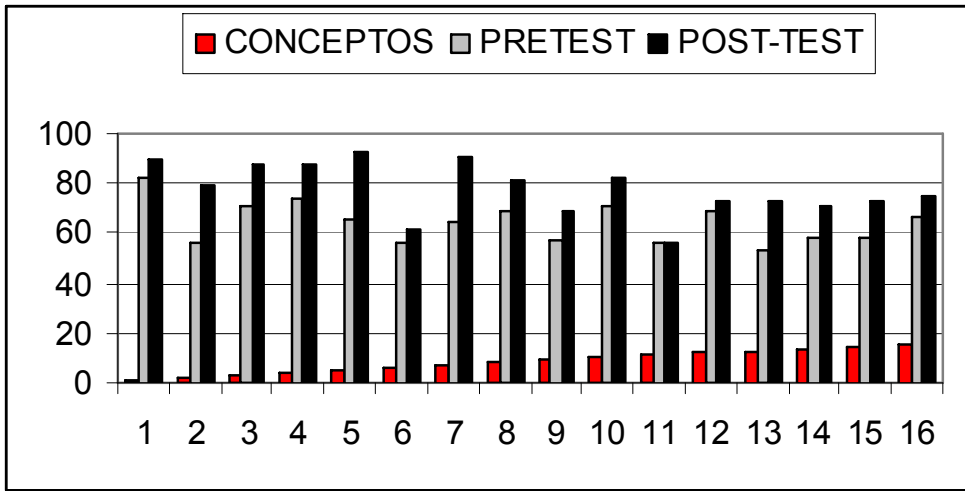

Gráfica 3. Diferencia de la estructura cognitiva

Tal como lo muestra la gráfica 3, todos los conceptos (a excepción del concepto dillución) fueron mejorados gracias a la estrategia didáctica empleada; los conceptos que experimentaron mayor resignificación fueron molaridad y pH con una diferencia del $26 \%$ mientras que el concepto de disolución no sufrió cambio alguno.

Prueba de hipótesis

Para demostrar que el aprendizaje significativo de los conceptos alcanzado por el grupo objeto de estudio se debe a la estrategia de la resolución de problemas, se emplea una prueba $Z$, la cual requiere los siguientes algoritmos (Martínez, 2006):

$$
\sigma\left(\overline{X_{1}}-\overline{X_{2}}\right)=\sqrt{\frac{\sigma_{1}^{2}}{N_{1}}+\frac{\sigma_{2}^{2}}{N_{2}}}
$$

Aplicando los valores calculados de desviación estándar y varianza del pretest y el post-test, se tiene que:

$$
\sigma\left(\overline{X_{1}}-\overline{X_{2}}\right)=\sqrt{\frac{75.30}{36}+\frac{32.69}{36}}=1.73
$$

Con este valor finalmente se calcula Z:

$$
Z=\frac{\bar{X}_{1}-\bar{X}_{2}}{\sigma\left(\bar{X}_{1}-\bar{X}_{2}\right)} \quad Z=\frac{62.14-51.31}{1.73}=6.26
$$

La ubicación de $Z$ en la curva normal se muestra en la gráfica 4:

Gráfica 4. Prueba de hipótesis

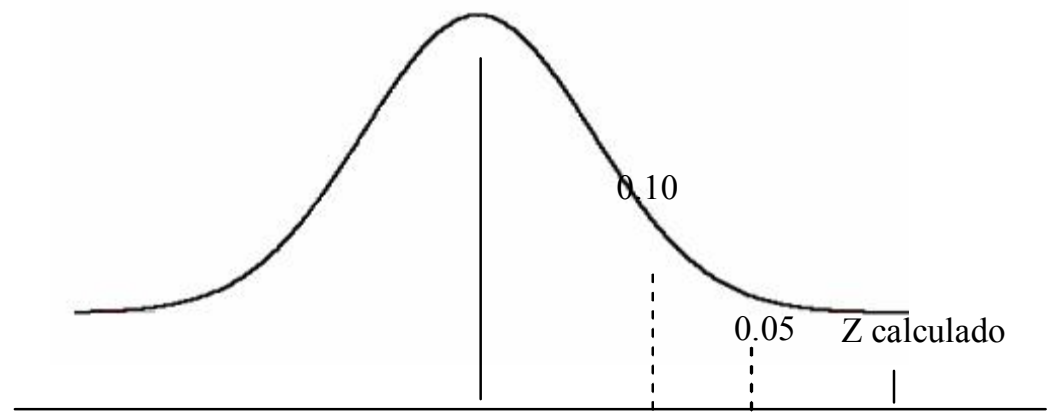

$$
\begin{array}{lll}
1.28 & 1.64 & 6.26
\end{array}
$$


El valor de $Z$ calculado se ubica en la zona de alta significancia de la curva normal con un intervalo de confianza menor a $\mathbf{0 . 0 0 5}$. En consecuencia se acepta la hipótesis alterna, es decir se demuestra que el aprendizaje significativo alcanzado depende de la estrategia didáctica de la resolución de problemas.

Análisis complementario: Proceso de resolución de problemas

A continuación se detallan en rasgos generales los principales aportes de cada grupo de estudiantes durante el proceso de su resolución; se hacen observaciones derivadas del análisis de los textos presentados por cada uno de los grupos y también se hacen transcripciones literales.

Primer problema. Para aliviar el dolor de cabeza, es frecuente el uso de aspirina efervescente. Si quieres resolver este tipo de problema de la manera más rápida y efectiva, utilizas ¿Agua caliente, al clima o fría?, ¿Utilizańas poca o bastante agua? Explica tus respuestas y justifícalas.

Solución: "la rapidez con que se disuelve la aspinina depende de la cantidad de agua y de su temperatura. Plantean que la mejor forma de disolver la aspirina es usando un volumen más 0 menos de unos 100 mililitros de agua a temperatura ambiente, en analogía a la concepción alternativa de que "al exponer algunos alimentos y medicamentos al frío, este ambiente hace que se conserve y no se degraden fácilmente, como sucedió cuando se agregó la aspirina al agua fría donde ésta no se disolvió totalmente".

Segundo problema. Intenta disolver azúcar en gasolina. Ahora intenta disolver glicerina en agua. Finalmente intenta disolver sal de cocina en alcohol antiséptico. Explicar y discutir los resultados de estas experiencias.

Solución: "no es posible disolver azúcar en gasolina por qué la gasolina es un compuesto orgánico no polar. La solubilidad de un disolvente está determinada en parte por la polaridad tanto del soluto como del solvente, y esta propiedad está a su vez derivada de la estructura molecular y las fuerzas de interacción, para que ocurra una disolución dichas características deben ser similares pues su efecto parecidas. Las dos experiencias adicionales no se realizaron.

Tercer problema. ¿Qué condiciones deben tener las sustancias para poder disolverse en otras?

Solución: El grupo realiza una práctica en la cual tratan de disolver varias sustancias caseras y otras de común uso en el laboratorio. Seleccionan bicarbonato de sodio, sal de cocina, azúcar, café liofilizado, gelatina en polvo, alcohol antiséptico, agua, aceite de cocina, blanqueador de ropa, detergente, al igual que algunos solventes comunes como etanol, acetona, acetaldehído y benceno. Las pruebas realizadas les permiten establecer que las sustancias polares se disuelven en sustancias polares y que las no polares lo hacen en sus similares.

Cuarto problema. ¿Cuáles son las unidades de concentración de soluciones químicas, más usadas a nivel mundial?

Las concepciones altemativas o preconcepciones del grupo de estudiantes les lleva a conduir de manera enfática que las unidades de concentración de las soluciones pertenecen a dos tipos: unidades químicas y unidades físicas. Dentro del primer grupo señalan a la molaridad y la normalidad como unidades universales de concentración, argumentando tener dificultades conceptuales para definir el concepto de equivalente gramo con el aual se calcula la normalidad. Dentro de las unidades físicas, demuestran manejar el porcentaje de solución (peso a peso, peso a volumen y volumen a volumen); sin embargo no pueden explicar la relación soluto a solvente para el caso de la concentración expresada en partes por millón, la cual está definida en miligramos de soluto por cada litro de disolución. El grupo difícilmente establece la relación que se establece al disolver un gramo de soluto en un metro cúbico de disolución, para deducir que en cada litro de ésta, se encuentra disuelto exactamente un miligramo de soluto. 
Quinto problema. Preparar una solución $0.5 \mathrm{~N}$ de $\mathrm{H}_{2} \mathrm{SO}_{4 \bullet,}$ luego diluirla a $0.025 \mathrm{M}$ y expresar esta concentración en p.p.m

Solución: A través de cálculos sencillos de dilución resuelven aritméticamente el problema (Gómez, 2007), sin embargo, el volumen final de la solución es enáticamente medido como volumen de agua requerido para efectuar la dilución. Los alumnos no pudieron transformar la concentración molar o normal en p.p.m. por considerar a éste último como un concepto nuevo, para el cual no pueden establecer poca o ninguna relación con sus conocimientos previos.

Sexto problema. Determinar el pH de los siguientes alimentos: leche, vino, jugo de naranja, tinto, CocaCola, suero oral, aceite de cocina, cerveza, vinagre.

El grupo de estudiantes efectúa la medición del pH de las bebidas anteriormente descritas, empleando papel indicador universal.

Por contrastación colorimétrica determinan los siguientes valores de pH“

\begin{tabular}{|l|l|l|l|l|l|l|l|l|l|}
\hline Sustancia & Leche & Vino & $\begin{array}{l}\text { Jugo } \\
\text { de } \\
\text { naranja }\end{array}$ & Tinto & $\begin{array}{l}\text { Coca- } \\
\text { Cola }\end{array}$ & $\begin{array}{l}\text { Suero } \\
\text { oral }\end{array}$ & $\begin{array}{l}\text { Aceite } \\
\text { de } \\
\text { cocina }\end{array}$ & Cerveza & Vinagre \\
\hline pH & $\mathbf{6}$ & $\mathbf{5}$ & $\mathbf{6}$ & $\mathbf{5}$ & $\mathbf{5}$ & $\mathbf{6}$ & $\mathbf{5}$ & $\mathbf{6}$ & $\mathbf{6}$ \\
\hline
\end{tabular}

De acuerdo con los datos de las bebidas anteriormente descritas, el grupo de estudiantes plantea la hipótesis de que todas las bebidas e induso los alimentos disueltos en agua, deben tener pH ácido.

Séptimo problema. Determinar las razones por las auales, los alimentos tienen pH ácido.

El grupo plantea varias razones para explicar el pH ácido de los alimentos, entre ellas se destacan:

a. El sistema de amortiguadores de pH del organismo se encuentra diseñado para trabajar óptimamente en medio ácido.

b. Consideran que el pH de los alimentos debe ayudar a los amortiguadores de pH del organismo que controlan los cambios de acidez producidos por el ejercicio muscular y por la ventilación pulmonar.

c. El metabolismo humano emplea enzimas que funcionan óptimamente a pH ácido.

Por otra parte pudieron identificar sin embargo que existen alimentos como el plátano y algunos granos que contienen componentes básicos.

Octavo problema. Al disolver $50 \mathrm{ml}$ de agua con $50 \mathrm{ml}$ de etanol absoluto $(96 \% \mathrm{v} / \mathrm{v})$, se espera preparar $100 \mathrm{ml}$ de disolución. Efectuar el procedimiento anteriormente descrito, explicar y analizar los resultados obtenidos. Adicionalmente plantear un modelo macroscópico que explique lo ocurnido.

Solución: Al mezclar los dos volúmenes el grupo mide un volumen final de $96 \mathrm{ml}$, argumenta que las moléculas del agua por tener menor tamaño en comparación con las moléculas de etanol, éste tiene la capacidad de filtrarse entre éstas ocupando sus espacios, concepción que se corresponde con la percepción sobre cambios químicos con un único modelo macroscópico de representación, planteado por Pozo y Gómez Crespo, 2005 y citados por Furió y Domínguez, 2007, donde el disolvente cede su lugar al soluto, (Gallegos y Garritz, 2004) para admitir la idea de vacío entre las moléculas implicadas (Gómez, Pozo y Gutiérrez, 2004) descartando la presencia de aire entre las mismas (Pozo y Gómez Crespo, 2004) como explicación lógica entre estudiantes de estas edades. 
Noveno problema. Plantear varias alternativas para utilizar productos naturales o de uso cotidiano, como indicadores de pH.

Solución: El grupo después de la indagación bibliográfica logra detectar que los vegetales coloreados de azul o rojo contienen pigmentos naturales, tales como las antocianinas, sensibles a los cambios de pH. Entre los vegetales con este tipo de característica, logran ubicar al repollo morado, los pétalos de rosas y daveles rojos y el jengibre, frutas y hortalizas como ejemplo las fresas, cerezas, ciruelas, cebolla cabezona roja, berenjena y la uva negra.

Para comprobar el hallazgo teórico deciden experimentar con hojas de repollo morado y pétalos de rosas, para conduir que efectivamente, los extractos de estas partes vegetales se tiñen de rojo en medio ácido y de azul en medio básico.

Décimo problema. Explicar lo que le ocurre a la pintura utilizada para pintar el exterior de los automóviles, al intentar disolverla con agua.

Solución: "el agua posee dos pares de electrones no compartidos que le permite formar puentes de hidrógeno con una atracción muy fuerte, a diferencia de la pintura a base de poliuretano que no aumple con esta propiedad y sus atracciones son muy débiles".

Las diferencias entre propiedades químicas derivadas de la estructura molecular genera la incompatibilidad de las partículas del soluto con las del solvente. Debido esta dificultad se comprueba la teoría que afirma que un compuesto similar disuelve a su similar.

\section{Conclusiones}

D El grupo objeto de estudio alcanza un aumento del $13.47 \%$ por encima de su punto de partida conceptual, grado de resignificación sustantivo si se considera que el grupo se inicia con un $62.40 \%$ de conocimiento del tema objeto de estudio y termina con un $76.67 \%$ del mismo. Aparentemente este porcentaje es pequeño pero como ya se señala, el grupo tiene un grado de conocimiento aceptable de los conocimientos pertenecientes a la unidad temática de soluciones químicas.

> El grado de resignificación alcanzado por el grupo objeto de estudio, demuestra que la estrategia constructivista de la resolución de problemas se consolida como una muy buena opción para lograr el aprendizaje significativo de los conceptos ligados a la temática de soluciones químicas. La resignificación lograda es el producto de la acción de resolver los problemas propuestos en el ejercicio investigativo sobre las preconcepciones del grupo. Los preconceptos son difíciles de cambiar y el desarrollo conceptual es un proceso lento, largo y difícil de alcanzar porque las concepciones cotidianas de los estudiantes están muy arraigadas; más aún, hay ausencia de publicaciones que demuestren que las concepciones altemativas hayan sido totalmente extinguidas y sustituidas por una nueva idea (Duit, 1999; Galili y Bar, 1992; Tao y Gunstone, 1999, citados por Galagovsky, 2007), como máximo se consigue que los alumnos lleguen a asimilar los conocimientos cient́ficos sin abandonar sus conocimientos cotidianos en la búsqueda de conocimiento, es decir, un acercamiento de la ciencia escolar a la ciencia erudita.

La resolución de problemas como estrategia didáctica facilita el trabajo colaborativo entre los grupos de trabajo, los obliga a apropiarse del método científico para establecer una secuencia de pasos conducentes hacia la definición de altemativas de solución pertinentes. La definición de los vacíos conceptuales, le permitió a cada grupo buscar información sobre su problema espeć́fico, dasificarla, aprehenderla resignificando sus ideas previas de una manera autónoma, derivada de la decisión personal y grupal para mejorar su saber espećfico. Igualmente le exige a cada grupo, la 
identificación y priorización de procedimientos y estrategias prácticas necesarias para abordar la solución de cada problema.

\section{Bibliografía}

Aiken, L. (2003). Estadística descriptiva. En: Test psicológicos y evaluación. Onceava edición. México: Pearson, Prentice Hall.

Ausubel, D. Novak, J. D. Hanesian, H. (1987). Psicología Educativa: un punto de vista cognoscitivo. Segunda edición. México:Trillas,

Ballester, A. (2002). El aprendizaje significativo en la práctica. Como hacer el aprendizaje significativo en el aula. Seminario de aprendizaje significativo. Palma de Mallorca. España.

Cohem, R, Swerdlik, M. (2001). Confiabilidad. En Pruebas y evaluación psicológicas: Introducción a las pruebas. Cuarta edición. (pp. 154 - 183). México: Mc Graw Hill.

Cohem, R, Swerdlik, M. (2001). Validez. En Pruebas y evaluación psicológicas: Introducción a las pruebas. Cuarta edición. (pp. 184 - 223). México: Mc Graw Hill.

Furió, C. Domínguez, C. (2007). Problemas históricos y dificultades de los estudiantes en la conceptualización de sustancia y compuesto químico. Enseñanza de las ciencias, 25(2), pp. 241258.

alagovsky, L (2007). Enseñanza versus aprendizaje de las ciencias naturales. Tecné, episteme $y$ didaxis, Número extraordinario. Tercer congreso intemacional sobre formación de profesores de ciencia. Bogotá. Colombia.

Gallegos, L. Garritz, A. (2004). Representación contínua y discreta de la materia en estudiantes de química. Educación química. 15(3). pp 234-242. Documento consultado el 15 de septiembre de 2007, en: http://uww.fquimunam.mx/sitio/edquim/153/153-gall.pdf

Gil, D. (1993). Los programas guía de actividades: una concreción del modelo constructivista de enseñanza y aprendizaje de las ciencias. Revista Investigación en la Escuela. Vol 8. № 3. Sevilla. España.

Giroux, S. Tremblay, G. (2004). Metodología de las Ciencias Humanas. La investigación en acción. México: Fondo de Cultura Económica.

Gómez, M.A. Pozo, J.I Gutiérrez, M.S. (2004). Enseñando a comprender la naturaleza de la materia: el diálogo entre la química y nuestros sentidos. Educación química. 15(3), pp.198-210. Documento consultado el 15 de septiembre de 2007, en:

http: //uww.fquim. unam.mx/sitio/edquim/153/153-poz.pdf

Gómez M. (2007). Factores que influyen en el éxito de los estudiantes al resolver problemas de química. Enseñanza de las ciencias. 25(1), 59-72

Hemández, R., Femández, C. Baptista, P. (2007). Metodología de la investigación. Cuarta edición. Iztapalapa, México D.F: McGraw-Hill.

Lozano, A. (2006). Diseño de programas educativos basados en competencias. Curso teórico práctico de la Maestría en Educación. Instituto tecnológico de Monterrey. México.

Martínez. C. (2005) Estadística y muestreo. Doceava edición. Santafé de Bogota, Colombia: Ecoe.

Perales, F. J. Álvarez, P. Femández, M. García. J.J. González, F. y Rivarrosa, A. (2000). Resolución de problemas. Madrid, España: Síntesis S.A.

Posner, G. Strike, K. Hewson, P. Gertzog, W. (1982). Accommodation of a scientific conception: Toward a theory of conceptual change. Saience Education: 66(2). 211-227Pozo, J. I., y Gómez, M. A. (2004). Aprender y enseñar ciencia. Del conocimiento cotidiano al conocimiento científica. Cuarta edición. Madrid, España: Morata. 


\section{Anexo 1. Cuestionario}

A continuación encontrarás una serie de afirmaciones numeradas del 1 al 16 , sobre las cuales te pedimos que definas tu opinión, usando los siguientes criterios: Totalmente de acuerdo, De cuerdo, Sin opinión, En desacuerdo, Totalmente en desacuerdo. Marca una $X$ en la casilla que consideres se corresponde con tu opinión personal:

Los resultados serán utilizados con propósitos exclusivamente investigativos, razón por la cual, tu nombre no va a ser usado bajo ninguna consideración, simplemente se utiliza como un mecanismo de control y seguimiento.
1. Una solución química es el producto de la relación que se establece entre dos o más sustancias miscibles (que se pueden mezclar) entre si.
Totalmente de acuerdo
De acuerdo
Sin opinión
En desacuerdo
Totalmente en desacuerdo

2. El soluto es la sustancia que se ha disuelto en el seno de un solvente y se encuentra en mayor proporción al interior de una solución.
Totalmente de acuerdo
De acuerdo
Sin opinión
En desacuerdo
Totalmente en desacuerdo

3. El solvente es la sustancia que permite la solubilidad del soluto y se encuentra en mayor proporción al interior de una solución química.

Totalmente de acuerdo

De acuerdo

Sin opinión

En desacuerdo

Totalmente en desacuerdo

4. Las moléculas polares son altamente solubles en agua
Totalmente de acuerdo
De acuerdo
Sin opinión
En desacuerdo
Totalmente en desacuerdo

5. La molaridad es una unidad de concentración química que expresa la cantidad de moles de soluto disueltas en un litro de solución.
Totalmente de acuerdo
De acuerdo
Sin opinión
En desacuerdo
Totalmente en desacuerdo

6. El cloro residual del agua potable tiene una concentración de 2 p.p.m.; eso significa que cada metro cúbico de agua tiene disueltos 2 miligramos de cloro en sus sales
Totalmente de acuerdo
De acuerdo
Sin opinión
En desacuerdo
Totalmente en desacuerdo

7. El pH de una solución se calcula aplicándole el logaritmo negativo a la concentración molar de iones de $\mathrm{H}^{+}$.
Totalmente de
De acuerdo
Sin opinión
En desacuerdo
Totalmente en acuerdo

8. Solamente las moléculas polares pueden disociarse en solución acuosa.
Totalmente de
De acuerdo
Sin opinión
En desacuerdo
Totalmente en
desacuerdo

9. Un electrolito débil es una sustancia cuyas moléculas tienen elevada polaridad y se disocian totalmente en solución acuosa.
Totalmente de
acuerdo
De acuerdo
Sin opinión
En desacuerdo
Totalmente en desacuerdo
10. El grado de solubilidad de las sustancias depende de su naturaleza química.
Totalmente de acuerdo
De acuerdo
Sin opinión
En desacuerdo
Totalmente en desacuerdo
11. Al diluir una solución de $\mathrm{HCl} 0.2 \mathrm{~N}$ hasta $\mathrm{HCl} 0.1 \mathrm{~N}$, la cantidad de soluto disuelto permanece constante.
Totalmente de acuerdo
De acuerdo
Sin opinión
En desacuerdo
Totalmente en desacuerdo

12. El grado de solubilidad de una sustancia es directamente proporcional a la temperatura de la solución en la cual se encuentre disuelto.
Totalmente de
De acuerdo
Sin opinión
En desacuerdo
Totalmente en acuerdo desacuerdo

13. Una solución con pH básico tiene una elevada concentración de iones $\mathrm{H}^{+}$.
Totalmente de
De acuerdo
Sin opinión
En desacuerdo
Totalmente en acuerdo desacuerdo

Las moléculas de los electrolito fuertes se disocian totalmente en solución acuosa.

Totalmente de acuerdo

De acuerdo

Sin opinión

En desacuerdo

Totalmente en desacuerdo

15. En las soluciones sobresaturadas, el solvente puede disolver el máximo de soluto posible.
Totalmente de
De acuerdo
Sin opinión
En desacuerdo
Totalmente en acuerdo desacuerdo

ica es un ejemplo de solución química.

Totalmente de acuerdo

De acuerdo

Sin opinión

En desacuerdo

Totalmente en desacuerdo 